
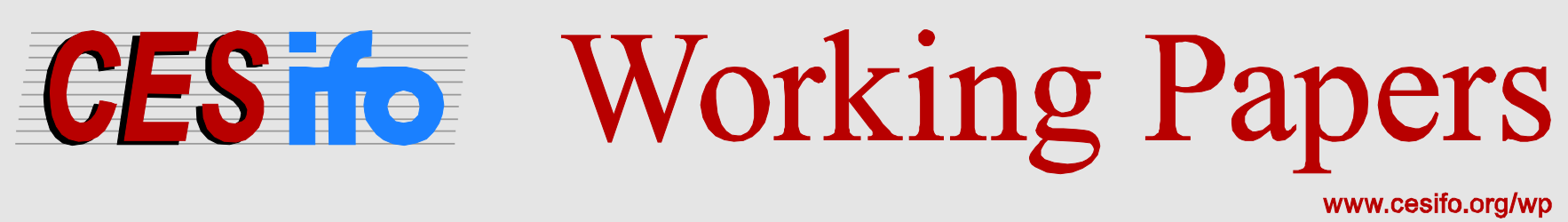

\title{
Reaching Market Equilibrium Merely by Bilateral Barters
}

\author{
Sjur Didrik Flåm
}

CESIFO WORKING PAPER NO. 4504

CATEGORY 13: BEHAVIOURAL ECONOMICS

DECEMBER 2013

\footnotetext{
An electronic version of the paper may be downloaded

- from the SSRN website:

- from the RePEc website:

- from the CESifo website:

www.SSRN.com

Www.RePEc.org

www.CESifo-group.org/wp
}

\section{CESifo}




\title{
Reaching Market Equilibrium Merely by Bilateral Barters
}

\begin{abstract}
Motivated by emission and resource markets, this paper considers repeated, bilateral barters between owners of commodity bundles, contingent claims, or property rights. Focus is on feasible, voluntary exchanges, driven only by differences in substitution rates. No coordination is ever needed. The modelling complies with adaptive learning, behavioral economics, and decentralized decision-making. Presuming transferable utility, the paper provides sufficient conditions for convergence to market equilibrium. It is expedient that some parties have differentiable objectives or make strictly feasible choices.
\end{abstract}

JEL-Code: C630, D030, D510.

Keywords: bilateral exchange, market equilibrium, transferable utility, common price, supergradients, stability, convergence.

\author{
Sjur Didrik Flåm \\ Economics Department \\ University of Bergen / Norway \\ sjur.flaam@econ.uib.no
}

November 28, 2013

Research supported by Finansmarkedsfondet, E.On Rhurgas, and the NILS exchange program. Thanks are due E. M. Ferragut and J. Tejada for discussions and Universidad Complutense de Madrid for hospitality. 


\section{INTRODUCTION}

The customary concept of Walrasian equilibrium presumes that agents are pricetaking maximizers. Thus, prices precede maximization. But where do prices come from? More to the point: is price-taking and maximization essential?

Confining attention to transferable utility, this paper suggests - as novelties - that

- prices need not come from anywhere - they may rather emerge;

- price-taking or maximization is neither necessary and nor quite realistic;

- agents could do without public prices;

- instead of maximizing, agents could seek improvements and avoid set-backs;

- direct exchanges can, by way of repeated deals, ensure convergence to equilibrium;

- everybody may contend with idiosyncratic, local information;

- no coordination, central agency, or global knowledge need ever be required.

Arguing for these assertions, this paper circumvents all causal or logical links, if any, between price-taking and maximization. In fact, either activity appears redundant - like a superfluous limitation of the solution concept. Most important: the process studied here below is fully driven by bilateral exchanges.

In a seminal paper, Feldman [9] also invoked just such exchanges. On so weak a premise, but presuming smooth objectives, he already showed that equilibrium may obtain. He required, however, that each deal yields a core outcome for the two trading parties. Thereby, optimization again takes center stage. Related studies include [20], [26], [38] and [42]. Many of these papers inquire whether Pareto optimality "in the small" suffices for efficiency "in the large."

This paper differs in orientation, being fully focused on possible emergence of efficiency and equilibrium. The approach is agent-based and computational [17], [18], [44], [45]. While inspired by behavioral and experimental economics [43], it contends with non-smooth data, and most important: no optimization - individual or joint is ever undertaken. Agents merely adapt, step by step, each move being somewhat moderate and myopic.

Still, the chief issues remain: can pair-wise, direct deals lead non-coordinated agents towards consistent choices? Is market equilibrium - whence Pareto efficiency - thereby attainable? ${ }^{1}$ Can agents proceed without public announcement of prices? Might common prices emerge as final outcomes, identifiable only after all desirable transactions are completed?

The paper addresses all these issues and provides positive answers. It relates to exchange economies, production games, and price-supported core solutions [13], [31]. Unlike most studies, it plays down the importance of agents' experience, information, rationality, and skills. Since real parties often are short on some among such desirable requisites, what appears below is a dynamic, but fictitious experiment - fully driven by somewhat short-sighted agents and by differences in their margins [43], [44]. Presumably, exchange goes on until no two agents can improve their lot. When stable,

\footnotetext{
${ }^{1}$ Several studies consider links between pairwise, t-wise, and overall Pareto optimality; see e.g. $[9],[10],[20],[26]$.
} 
the resulting process provides some micro-foundation for market equilibrium.

Numerous studies have offered various underpinnings of such equilibrium. While emphasizing strategic aspects, many deal with anonymous trading posts, or merely with two-sided markets - or with indivisible goods to be transferred from sellers to buyers [37]. In one line of inquiry, agents meet pair-wise, maybe randomly, to bargain [32], [38]. Other approaches evoke auctions or iterative price mechanisms [17], [40].

In contrast, the market depicted below is not composed of pure buyers and sellers; diverse parties may demand some goods, but supply others. Goods are divisible, transferable, and possibly numerous. Transactions could occur in fixed, periodic, or random order. Bargaining or haggling is not precluded, but never made explicit. No auctions, market makers, or price mechanisms need ever come into play.

At no intermediate stage must choices be efficient, optimal, or stable somehow. In fact, agents can come forwards with moderate competence, fairly general feasible sets, and non-smooth objectives. Nobody must display optimizing or forward-looking capacities. And nobody need ever develop behavioral strategies. In short, exchange can proceed without perfection in competence, foresight, or rationality.

The setting may fit exchange of natural resources or user-rights to such. Important instances comprise transfers of fish quotas, production allowances, pollution permits, or rights to water usage. One may envisage that public-private enterprises coexist with governmental agencies, the latter acting as managers or owners of commons.

Also fitting is trade of contingent claims [11]. In that case, what comes up is mutual insurance or security exchange [12]. Markets for such items are well developed. In contrast, large investment projects are less marketable. Yet, if construed as members of a portfolio, the value of each can be studied via the approach adopted below [15].

The paper adds to and extends recent studies [12], [13], [14], [15]. Its analytical innovations come by allowing simple non-optimal steps, preserving feasibility throughout, and permitting non-smooth data. Its economic novelties amount to show that decentralized, direct, two-sided deals may generate clearing prices. The attending convergence result speaks for the stability of equilibrium in stationary economies as well as for the expediency of bilateral barters. The size of the economy appears unimportant. Despite small numbers, the likelihood of equilibrium emergence seems non-negligible.

In the sequel, the role of convexity is somewhat reduced. Its presence will be emphasized precisely when and where crucial. Likewise, but more on a technical note, classical differentiability is largely dispensed with. Only for a final step, from pair-wise to overall efficiency, at least one agent should make a smooth and strictly feasible choice. ${ }^{2}$ Also on this point, paper adds the received literature which stresses the advantages of smooth criteria across the entire agent set and either all agents holding some common commodity or some agent holding all commodities [9]. To bring all this out, the paper is planned as follows.

\footnotetext{
${ }^{2}$ This weakens the differentiability conditions in [9].
} 
Section 2 introduces the setting, defines solutions, and characterizes these.

Section 3 models exchange as direct, between only two parties at a time.

Section 4 studies convergence to market equilibrium.

Section 5 relates direct exchange to differences in gradients and margins.

Section 6 outlines three examples.

Section 7 concludes. Proofs are deferred to appendix.

\section{The Setting and Equilibrium}

This section introduces the chief components of the model as well as some preliminaries.

Consider a fixed, finite set $I$ of economic agents, all engaged in exchange of vectors that belong to a finite-dimensional space $\mathbb{X}$, equipped with inner product $\langle\cdot, \cdot\rangle$ and associated norm $\|\cdot\| \cdot{ }^{3}$ Each vector in $\mathbb{X}$ represents a commodity bundle, contingent claim, or property rights.

Agent $i \in I$ already holds endowment $e_{i} \in \mathbb{X}$. When possible, he seeks to improve own payoff $u_{i}\left(x_{i}\right) \in \mathbb{R}$ by way of repeated bilateral exchanges. His choice $x_{i}$ must always belong to a non-empty closed set $X_{i} \subseteq \mathbb{X}$.

Besides the goods that form bundles in $\mathbb{X}$ there is another good, construed as transferable utility, money, or pecuniary reward, serving as means of payment [41]. Thus, agent $i$ worships maximization of an objective

$$
\left(x_{i}, r_{i}\right) \in X_{i} \times \mathbb{R} \mapsto u_{i}\left(x_{i}\right)+r_{i} \in \mathbb{R}
$$

commonly referred to as quasi-linear utility. His initial monetary wealth is of no consequence for subsequent choices, and income that results from trade, is unconstrained throughout. ${ }^{4}$

For interpretation, one may regard $u_{i}\left(x_{i}\right)$ as the profit of a producer who implements input-output bundle $x_{i}$. Alternatively, $u_{i}$ is a production function for one output sold at unit price.

A profile $\mathbf{x}=\left(x_{i}\right) \in \mathbb{X}^{I}$ is called an allocation iff $\sum_{i \in I} x_{i}=\sum_{i \in I} e_{i}=: e_{I}$. It is feasible moreover, iff $x_{i} \in X_{i}$ for each $i$. A feasible allocation is declared efficient provided it realizes the optimal value

$$
u_{I}(x):=\sup \left\{\sum_{i \in I} u_{i}\left(x_{i}\right) \mid \sum_{i \in I} x_{i}=x \text { and } x_{i} \in X_{i}\right\}
$$

when the aggregate endowment $x=e_{I}$. Naturally assume that $u_{I}\left(e_{I}\right)$ be finite. ${ }^{5}$

The main concerns here are with fully integrated market equilibrium and its possible attainment merely via repeated bilateral exchanges. So, by tacit assumption,

\footnotetext{
${ }^{3}$ The subsequent arguments and notations allow consideration of Hilbert space settings.

${ }^{4}$ Of course, no agent, present here, can create money out of nothing. Otherwise the concept of transferable utility would become elusive.

${ }^{5}$ By convention, in (1) and elsewhere, $\sup \emptyset=-\infty$.
} 
no auctioneer or referee will come to the fore - and no central planner, coordinator, or invisible hand will work backstage.

Definition (Market equilibrium). A feasible allocation $\mathbf{x}=\left(x_{i}\right)$, alongside a price vector $p \in \mathbb{X}$, constitutes a market equilibrium iff

$$
u_{i}\left(x_{i}\right)+\left\langle p, e_{i}-x_{i}\right\rangle \geq u_{i}(\chi)+\left\langle p, e_{i}-\chi\right\rangle \text { for each } i \in I \text { and } \chi \in X_{i} .
$$

In essence, subject to non-strategic behavior, demand should equal supply when everybody maximizes own payoff plus the value of his net sales. Thus, the solution concept - and the final outcome - is market clearing in a production-exchange economy where utility and endowments are perfectly transferable.

The definition requires merely two things: first, the consistency of individuals' plans; second, no single agent can improve his choice at equilibrium prices. Neither price-taking behavior nor complete markets is presumed. Thus, we avoid some selfimposed limitations embedded in the standard concept of Walrasian equilibrium. Accordingly, no price curves come up here. And no mention is made of infinitely elastic demand schedules - so prominent in perfect competition.

The cardinality of $I$ plays no role; there is no concern with the size of the economy or the effectivity of small groups [46]. The no-surplus condition, which characterizes perfect competition [28], [33] and the marginal productivity theory of distribution [34], does not hold; see Example 2.3.

I envisage that $u_{i}\left(x_{i}\right)$ emerges as the best monetary value of a tradeable inputoutput vector $x_{i}$; confer Example 6.2. ${ }^{6}$ This optic partly justifies the consideration of transferable utility and resources.

Of chief interest are various forms of dynamics. Clearly, iterated adaptations of endowments precede the arrival, if ever, at a steady state - if any. So, the dynamic phase deserves priority. Nonetheless, for a long perspective, I pause briefly to discuss characterization and existence of equilibrium. To this end, recall that $x^{*} \in \mathbb{X}$ is a supergradient of a function $f: \mathbb{X} \rightarrow \mathbb{R} \cup\{-\infty\}$ at $x \in \mathbb{X}$, as signalled by writing $x^{*} \in \partial f(x)$, iff $f(x)$ is finite and

$$
f(\chi) \leq f(x)+\left\langle x^{*}, \chi-x\right\rangle \text { for each } \chi \in \mathbb{X} .
$$

With reference to (1), any $p \in \partial u_{I}\left(e_{I}\right)$ is henceforth named a shadow price. No convexity assumption is invoked here. Yet, shadow prices capture the essence of solutions:

Theorem 2.1 (Characterization and existence of equilibrium).

- (On shadow pricing and equal margins). Let the function $\check{u}_{i}: \mathbb{X} \rightarrow \mathbb{R} \cup\{-\infty\}$ equal $u_{i}$ on $X_{i}$ and $-\infty$ elsewhere. Then, for any efficient allocation $\left(x_{i}\right)$,

$$
p \in \partial u_{I}\left(e_{I}\right) \Leftrightarrow p \in \partial \check{u}_{i}\left(x_{i}\right) \text { for each } i .
$$

\footnotetext{
${ }^{6}$ For precisely that reason some reduced criterion $u_{i}(\cdot)$ could come non-differentiable at places; see Example 6.2.
} 
- (On equilibrium). Any efficient allocation alongside any shadow price constitute an equilibrium. Conversely, every equilibrium allocation is efficient, and every associated price must be a corresponding shadow entity.

- (Existence of an efficient allocation). Assume each $u_{i}$ upper semicontinuous. Suppose there is a subset $\mathcal{I} \subseteq I$ such that $X_{i}$ is bounded when $i \in \mathcal{I}$, and $\sum_{i \notin \mathcal{I}} u_{i}\left(x_{i}\right) \rightarrow$ $-\infty$ as $\left\|\left(x_{i}\right)_{i \notin \mathcal{I}}\right\| \rightarrow \infty$. Then there exists an efficient allocation.

- (Existence of a shadow price). Let $\hat{u}_{I}: \mathbb{X} \rightarrow \mathbb{R} \cup\{-\infty\}$ denote the smallest concave function pointwise $\geq u_{I}$, the latter being defined in (1). If $\partial \hat{u}_{I}\left(e_{I}\right)$ is non-empty and $\hat{u}_{I}\left(e_{I}\right)=u_{I}\left(e_{I}\right)$, then each $p \in \partial \hat{u}_{I}\left(e_{I}\right)$ is a shadow price.

Example 2.1 (Smoothness isn't needed). Let $\mathbb{X}=X_{i}=\mathbb{R}$, each $u_{i}\left(x_{i}\right)=\min \left\{x_{i}, 1\right\}$, and $e_{i} \in(0,1)$ with $\sum_{i \in I} e_{i}=1$. Allocating the entire aggregate endowment to one agent is efficient, and $p=1$ becomes an equilibrium price. Everybody takes home payoff $p e_{i} . \diamond$

Example 2.2 (Concavity and continuity is not necessary). The same conclusions as in Example 2.1 hold with

$$
u_{i}\left(x_{i}\right):=\left\{\begin{array}{cl}
x_{i} & \text { when } x_{i} \leq 0 \\
0 & \text { when } x_{i} \in(0,1) \\
1 & \text { elsewhere. }
\end{array}\right.
$$

Example 2.3 (Equilibrium with surplus). Let $\mathbb{X}=\mathbb{R}$ and $X_{i}=\mathbb{R}_{+}$. Suppose a single land-lord $0 \in I$ derives concave, strictly increasing payoff $u_{0}\left(x_{0}\right)$ from labor input $x_{0}$, with $u_{0}(0)=0=e_{0}$. Each agent $i \neq 0$ is a landless worker, so that $u_{i} \equiv 0$, but he can deliver effort $e_{i} \geq 0$. Then, provided $e_{I}>0$, any $p \in \partial u_{0}\left(e_{I}\right)$ becomes an equilibrium price, and $\mathbf{x}=\left(e_{I}, 0, \ldots, 0\right)$ is the unique efficient allocation. The presence of the landlord benefits each worker [33]. $\diamond$

Theorem 2.1 invites a traditional, top-down perspective. It starts from coordinated shadow pricing at the upper, system level and descends down to equal margins across agents. As noted, in such a perspective, no function $-u_{i}$ or set $X_{i}$ need be convex. ${ }^{7}$

This paper approaches matters from the opposite end. Starting from unequal margins deep down, it moves upwards, by way of bilateral barters, to consider possible emergence of common pricing. Upon travelling this turned-around path, convexity comes handy at places. To indicate how, recall that for any non-empty set $X \subseteq \mathbb{X}$,

$$
N_{X}(x):=\left\{x^{*} \in \mathbb{X} \mid\left\langle x^{*}, \chi-x\right\rangle \leq 0 \text { for all } \chi \in X\right\}
$$

is named its outward normal cone at $x \in X$. Granted convexity, such cones $N_{i}:=N_{X_{i}}$ help in describing efficiency:

\footnotetext{
${ }^{7}$ What imports is only that $-u_{I}$ be convex with respect to one point, namely $e_{I}$.
} 
Corollary 2.1 (Equilibrium and one price). For each $i$, suppose $-u_{i}$ and $X_{i}$ are convex. In that case, at any allocation $\mathrm{x}=\left(x_{i}\right)$, if all agents see a common price

$$
p \in \cap_{i \in I}\left[\partial u_{i}\left(x_{i}\right)-N_{i}\left(x_{i}\right)\right]
$$

then $(\mathbf{x}, p)$ is an equilibrium. Conversely, if $(\mathbf{x}, p)$ is an equilibrium, (2) holds, and $p \in \partial u_{I}\left(e_{I}\right)$.

Example 2.4 (An incomplete security market). Suppose $X_{i}$ is a linear subspace of $\mathbb{X}=\mathbb{R}^{S}, S$ being a finite set of states. Then, with $u_{i}$ concave and differentiable, (2) says that $p$ equals the projection of the gradient $u_{i}^{\prime}\left(x_{i}\right)$ onto $X_{i}$. Agent $i$ can, by his market behavior, only reveal the $X_{i}$-component of his gradient; the part that resides in the orthogonal complement $X_{i}^{\perp}=N_{i}\left(x_{i}\right)$ remains hidden; see [27] and Example 6.3. below. $\diamond$

The preceding results indicate the convenience of having each $X_{i}$ convex and each $u_{i}$ concave. Such assumptions will, however, wait until they are needed.

So far, efficiency was described by a dual approach - via prices. An alternative, primal perspective, which invokes feasible directions and displacements, is also expedient - as shown next.

Given a subset $X \subseteq \mathbb{X}$ and point $x \in X$, let

$$
D_{X}(x):=\{d \in \mathbb{X} \mid x+r d \in X \text { for all } r>0 \text { sufficiently small }\}
$$

denote the cone of feasible directions. For any function $f: \mathbb{X} \rightarrow \mathbb{R} \cup\{-\infty\}$, and point $x \in \mathbb{X}$ where $f(x)$ is finite, let

$$
f^{-}(x ; d):=\lim \inf _{r \rightarrow 0^{+}} \frac{f(x+r d)-f(x)}{r}
$$

denote the lower derivative in direction $d \in \mathbb{X}$. In these terms, writing $D_{i}:=D_{X_{i}}$, efficiency shows up in the form of a variational inequality (4):

Proposition 2.2 (Efficiency and unprofitable deviations). Consider a feasible allocation $\left(x_{i}\right)$. If efficient, then

$$
\sum_{i \in I} u_{i}^{-}\left(x_{i} ; d_{i}\right) \leq 0 \text { for all } d_{i} \in D_{i}\left(x_{i}\right) \text { such that } \sum_{i \in I} d_{i}=0 .
$$

Conversely, if each $-u_{i}$ and $X_{i}$ is convex, (4) suffices for efficiency.

I henceforth take existence for granted in order to inquire: Can equilibrium come about merely by bilateral barters? Can agents proceed without prices ever being elicited? 


\section{Bilateral Barter}

By assumption, all dynamics derive from bilateral barters [24], [45]. It's appropriate therefore to isolate a single barter from others. So, this section considers one episode of the following recurrent sort: Two agents $i, j \in I$ meet, then holding respective bundles $x_{i} \in X_{i}$ and $x_{j} \in X_{j}$. On such an occasion they could, in principle,

$$
\text { maximize } u_{i}\left(x_{i}^{+1}\right)+u_{j}\left(x_{j}^{+1}\right) \text { s. t. }\left(x_{i}^{+1}, x_{j}^{+1}\right) \in X_{i} \times X_{j} \& x_{i}^{+1}+x_{j}^{+1}=x_{i}+x_{j} .
$$

Any optimal values $u_{i}\left(x_{i}^{+1}\right), u_{j}\left(x_{j}^{+1}\right)$ would ensure Pareto efficiency. Besides such efficiency, either party might also demand rationality, meaning

$$
u_{i}\left(x_{i}^{+1}\right) \geq u_{i}\left(x_{i}\right) \quad \& \quad u_{j}\left(x_{j}^{+1}\right) \geq u_{j}\left(x_{j}\right) .
$$

Each pair $\left(x_{i}^{+1}, x_{j}^{+1}\right)$ which solves (5) subject to (6) generates a core solution to the two-person, cooperative game at hand. Like Feldman [9] one may insist that agents $i, j$ indeed implement a core deal. I hesitate, however, in presuming so much. The reasons are several:

* Resolution of problem (5) could easily overstretch the competence or diligence of either party [17].

* Real agents rather seldom prove expert negotiators or optimizers.

* Exact resolution of (5) requires that constraints and objectives be fully revealed.

* It is likely that each agent only sees a local, linearized version of his decision problem. Typically, he lacks a global perception of the setting. At his actual holding, he might merely discern a "payoff gradient" as well as a conical approximation to the feasible set.

* Inequalities (6) reflect that utility isn't transferable. Such is not the setting here.

In short, for greater flexibility and realism, suppose the interlocutors act with moderate competence and some myopia. To formalize how, note that any updated holding $x_{i}^{+1} \neq x_{i}$ of agent $i$ defines a unit direction $d:=\left(x_{i}^{+1}-x_{i}\right) /\left\|x_{i}^{+1}-x_{i}\right\|$ of transfer to $i$ from $j$ - and a positive step-size $\sigma:=\left\|x_{i}^{+1}-x_{i}\right\|$ along that direction. Thus, presuming that direct exchange neither creates nor destroys any amount of any commodity, there is no loss of generality in viewing the process unfold as repeated manifestations of

$$
x_{i}^{+1}:=x_{i}+\sigma d \in X_{i} \quad \text { and } \quad x_{j}^{+1}:=x_{j}-\sigma d \in X_{j},
$$

with $\sigma>0$ and $\|d\|=1$.

The first inclusion in (7) tells that $d$ had better belong to the cone $D_{i}\left(x_{i}\right)=$ $D_{X_{i}}\left(x_{i}\right)$; see $(3)$. Likewise, $-d$ should reside in $D_{j}\left(x_{j}\right)$. So, it's indispensable that

$$
d \in D_{i j}\left(x_{i}, x_{j}\right):=D_{i}\left(x_{i}\right) \cap-D_{j}\left(x_{j}\right) .
$$

When several directions are feasible, which appear attractive? Clearly, if the aggregate holding $x_{i}+x_{j}$ permits strict improvement over $u_{i}\left(x_{i}\right)+u_{j}\left(x_{j}\right)=: u_{i j}\left(x_{i}, x_{j}\right)$, the agents shouldn't miss such a chance. The increment in their joint payoff

$$
\Delta u_{i j}=\Delta u_{i j}\left(x_{i}, x_{j}, \sigma, d\right):=u_{i}\left(x_{i}+\sigma d\right)+u_{j}\left(x_{j}-\sigma d\right)-u_{i}\left(x_{i}\right)-u_{j}\left(x_{j}\right)
$$


ought then be notable. To clarify that concern of theirs, it's natural to invoke the concept of steepest slope. Henceforth suppose the directional derivative

$$
\mathfrak{S}_{i j}\left(x_{i}, x_{j}, d\right):=\lim _{\sigma \rightarrow 0^{+}} \frac{\Delta u_{i j}\left(x_{i}, x_{j}, \sigma, d\right)}{\sigma}
$$

is well defined for any two agents $i, j \in I$ at each pair $\left(x_{i}, x_{j}\right) \in X_{i} \times X_{j}$ when $d \in D_{i j}\left(x_{i}, x_{j}\right)$.

Definition 3.1 (The steepest slope). At $\left(x_{i}, x_{j}\right) \in X_{i} \times X_{j}$ the joint payoff $u_{i}+u_{j}$ to agents $i, j$ has steepest slope

$$
\mathfrak{S}_{i j}\left(x_{i}, x_{j}\right):=\sup \left\{\mathfrak{S}_{i j}\left(x_{i}, x_{j}, d\right) \mid d \in D_{i j}\left(x_{i}, x_{j}\right) \&\|d\| \leq 1\right\} .
$$

Section 5 invokes convexity to study this slope in detail. For now, this entity only serves as a benchmark.

Any $d$ that realizes the supremum in (10) qualifies as a best direction. Admittedly, it may require some effort or skill of agents $i, j$ to identify the said slope - and to find an associated best direction, if any. ${ }^{8}$ So, if these objects were to play important roles, their demanding nature could frustrate agents who show moderate enthusiasm or excellence in computation. Put differently: assigning prominence to the steepest slope appears at variance with this paper's basic purpose - which is to provide a low-complexity model of agent-based barters. These queries motivate a more relaxed notion, one for which (10) is realized at least up to a fixed fraction $\varphi_{i j} \in(0,1)$ :

Definition 3.2 (Real transfer). Agents $i, j$ are said to make a real transfer if (7) holds with $d \in D_{i j}\left(x_{i}, x_{j}\right),\|d\|=1$, and

$$
\Delta u_{i j} \geq \sigma \varphi_{i j} \mathfrak{S}_{i j}\left(x_{i}, x_{j}\right)>0 .
$$

Proposition 3.1 (On real transfers). Whenever $\left(x_{i}, x_{j}\right) \in X_{i} \times X_{j}$ and $\mathfrak{S}_{i j}\left(x_{i}, x_{j}\right)>$ 0 , agents $i, j$ may indeed make a real transfer.

A real transfer (11) ensures, of course, that $\Delta u_{i j}>0$. Then, the inequalities

$$
u_{i}\left(x_{i}^{+1}\right)+\Delta r_{i}>u_{i}\left(x_{i}\right) \text { and } u_{j}\left(x_{j}^{+1}\right)+\Delta r_{j}>u_{j}\left(x_{j}\right)
$$

are solvable with monetary side-payments or "rewards" $\Delta r_{i}, \Delta r_{j}$ that sum to zero; see Example 6.1. Indeed, if only $u_{i}\left(x_{i}^{+1}\right)>u_{i}\left(x_{i}\right)$, offer $j$ additional payment $\Delta r_{j}$ such that $u_{j}\left(x_{j}\right)-u_{j}\left(x_{j}^{+1}\right)<\Delta r_{j}<u_{i}\left(x_{i}^{+1}\right)-u_{i}\left(x_{i}\right)$ and debit $i$ that same amount.

\footnotetext{
${ }^{8}$ Quite often, however, the best direction comes up immediately, at moderate computational cost - or after little cognitive effort. Specifically, this happens when:

- each feasible set $X_{i}$ is a non-negative orthant; conf. [9] and Example 6.1 below;

- each function $u_{i}$ derives as the optimal value from linear or concave optimization; see Example

6.2 on production games;

- each $X_{i}$ is a subspace, as illustrated by Example 6.3 on asymmetric information [11].
} 
Thus, real transfers can proceed by way of strictly Pareto improving steps - a most natural condition. Put differently: money "oils" the transaction machinery. Deals and incentives are compatible.

Nothing will be said, however, about how agents $i, j$ divide $\Delta u_{i j}>0$. As explained above, there is room for some satisfaction of either party. Fehr and Schmidt [8] report evidence that people do not exploit their power in bilateral bargaining. Andreoni and Bernheim [1] explain this phenomenon in terms of social images. Whatever be the explanations, outcomes, and theories of bargaining [32], [38], those issues are deliberately left open here.

A closer study of best directions is deferred to Section 5. The choice of appropriate step sizes is discussed next - as part of the subsequent convergence analysis.

\section{Convergence to Market Equilibrium}

This section considers whether repeated, bilateral barters converge. Just for the sake of organizing the arguments, it's expedient to view the exchange process unfold like an algorithm, fictitious or real, but affected by some protocol that decides who will trade next with whom.

\section{Repeated bilateral barters construed as an algorithm:}

- Start with some feasible allocation $\mathbf{x}=\left(x_{i}\right)$.

- Invoke the protocol to activate a novel agent pair $i, j \in I$.

- If $\mathfrak{S}_{i j}\left(x_{i}, x_{j}\right)=0$, invoke the protocol anew. Stop only when all steepest slopes vanish.

- Otherwise, $i, j$ make a real transfer (11).

- Continue to invoke the protocol until convergence.

Thus, at discrete stages $k=0,1, \ldots$. two selected agents make a real transfer. Like any algorithm, the above schematic outline invites queries about stopping, protocols, and convergence. These issues are addressed next, the first two only briefly.

Stopping is idealized and too stringent here. In practice, exchange terminates, and the market settles, when all $\mathfrak{S}_{i j}\left(x_{i}, x_{j}\right)$ are negligible or so small as to pass unnoticed. Then, no additional deals are worth any agent's while.

Protocols can be manifold. There is room for random pairing, deliberate search, asynchronous or parallel matching - and for different affinities among agents. Broadly, what imports is that each agent pair be activated repeatedly - or only when their steepest slope is largest across the set of player pairs. Here, for lack of space, I shall only consider two protocols:

In a first, encounters are quasi cyclic. This means that each agent pair meets at least once during every time interval of some fixed, finite length $l$.

In a second, periodically, the pair that trades enjoys maximal steepest slope. 
Convergence is more delicate and the main concern. Ultimately, a common price vector should emerge which balance the market. However, to begin with, it's prudent to contend with less, namely: all slopes should finally vanish. Broadly, this means that, in the end, all prospects for bilateral improvements are exhausted.

To secure such a weak sort of convergence, I first impose quasi-cyclic protocol. During its execution, step-sizes should dwindle, meaning

$$
\sigma^{k} \rightarrow 0^{+}
$$

yet, not too fast. To wit, for any subsequence $K \subseteq\{0,1, \ldots\}$, along which some agent pair $i, j$ make real transfers, it should hold that

$$
\lim _{k \in K}\left(x_{i}^{k}, x_{j}^{k}\right)=\left(x_{i}, x_{j}\right) \& \quad \lim \inf _{k \in K} \mathfrak{S}_{i j}\left(x_{i}^{k}, x_{j}^{k}\right)>0 \Rightarrow \sum_{k \in K} \sigma^{k}=+\infty .
$$

(12) reflects learning, satisficing behavior, market maturation, and eventual reduction in volatility. (13) requires that agents $i, j$ who trade and see convergence along a subsequence, with $\mathfrak{S}_{i j}$ bounded away from zero, shouldn't steadily forego possible improvements. Put differently: agents attend to good options - and respond to proper incentives with notable drive. (Section 5 concludes with a remark on (13)).

In short, conditions (12), (13) appear reasonable. Either only concerns asymptotics. In the interim agents can enjoy great freedom; they may apply maximizing steps or inaccurate line search. And finite-time convergence isn't excluded.

Proposition 4.1 (On exhaustion of two-sided trade options). Suppose each steepest slope $\mathfrak{S}_{i j}$ is lower semicontinuous on $X_{i} \times X_{j}$. Let encounters be quasi cyclic, transfers real, and step-sizes dwindling, but not too fast. Then, at every accumulation point of the resulting sequence $\mathbf{x}^{k}=\left(x_{i}^{k}\right)$ all steepest slopes are nil.

It's interesting to weaken the requirement (11). Broadly, one could contend with $\max _{i, j} \mathfrak{S}_{i j}>0 \Rightarrow \max _{i, j} \Delta u_{i j}>0$. Along that line, let the protocol periodically activate an agent pair which enjoys maximal $\mathfrak{S}_{i j}$. What happens at other stages is left unspecified. But clearly, feasibility had better be maintained throughout, and overall payoff $\sum_{i \in I} u_{i}$ should steadily increase.

Proposition 4.2 (More on exhaustion of two-sided trade options). Suppose that periodically, agents with maximal $\mathfrak{S}_{i j}$ make real transfers. Also suppose trading agents $i, j$ use a step-size $\sigma \geq \underline{\sigma}_{i j}\left(x_{i}, x_{j}\right)$ where $\underline{\sigma}_{i j}$ is lower semicontinuous and positive wherever $\mathfrak{S}_{i j}\left(x_{i}, x_{j}\right)>0$. Then, provided each $u_{i}$ is upper semi-continuous, all steepest slopes are nil at every accumulation point of the resulting sequence.

Propositions $4.1 \& 2$ dealt with convergence to the set

$$
\mathbf{A}_{0}:=\left\{\text { feasible allocation } \mathbf{x} \mid \text { all } \mathfrak{S}_{i j}\left(x_{i}, x_{j}\right)=0\right\} .
$$


The aim is, however, to reach the set $\mathbf{E}$ of equilibrium allocations. Clearly, $\mathbf{E} \subseteq \mathbf{A}_{0}$, but alas this inclusion can be strict:

Example 4.1 (Complete trade with no common price). Consider three agents $i \in$ $\{1,2,3\}$ and two goods $s \in S=\{1,2\}$. Posit $\mathbb{X}=\mathbb{R}^{S}$, write $x_{i s}:=x_{i}(s)$, and use concave utility functions

$$
\begin{aligned}
& u_{1}\left(x_{1}\right)=\min \left\{0, x_{11}\right\} \\
& u_{2}\left(x_{2}\right)=\min \left\{0, x_{22}\right\} \\
& u_{3}\left(x_{3}\right)=\frac{1}{2}\left[\min \left\{0, x_{31}, x_{32}\right\}+x_{31}+x_{32}\right]
\end{aligned}
$$

Let choice be unconstrained: $X_{i}=\mathbb{X}$. Consequently, each normal cone $N_{i}\left(x_{i}\right)$ is degenerate everywhere, containing only $0:=(0,0)$. At $x_{i}=e_{i}:=\mathbf{0}$ the agents have superdifferentials

$$
\partial u_{1}\left(x_{1}\right)=[\mathbf{0},(1,0)], \quad \partial u_{2}\left(x_{2}\right)=[\mathbf{0},(0,1)], \quad \partial u_{3}\left(x_{3}\right)=\operatorname{conv}\left\{\left(\frac{1}{2}, \frac{1}{2}\right),(1,0),(0,1)\right\} .
$$

Consequently, $\partial u_{i}\left(x_{i}\right) \cap \partial u_{j}\left(x_{j}\right) \neq \emptyset$ for each agent pair $i, j$ - that is, each $\mathfrak{S}_{i j}\left(x_{i}, x_{j}\right)=$ 0 so that $\mathbf{x} \in \mathbf{A}_{0}$ - but $\mathbf{x} \notin \mathbf{E}$ because $\cap_{i \in I} \partial u_{i}\left(x_{i}\right)$ is empty. $\diamond$

Presence of at least two commodities was essential in Example 4.1. With merely one commodity available, equilibrium obtains immediately:

Proposition 4.3 (Equilibrium with one commodity ${ }^{9}$ ). Suppose the commodity space is one-dimensional: $\mathbb{X}=\mathbb{R}$. Let each function $-u_{i}$ and set $X_{i}$ be convex. Then, any feasible allocation $\left(x_{i}\right)$ at which all steepest slopes are nil is also an equilibrium.

Besides the complete absence of constraints, it also distinguishes Example 4.1 that each function $u_{i}$ is non-smooth. If merely one agent has a differentiable unconstrained objective, equilibrium easily obtains. More generally, it follows forthwith

Proposition 4.4 (Single-agent smoothness and equilibrium). Suppose each function $-u_{i}$ and set $X_{i}$ is convex. Also suppose that at every $\mathbf{x} \in \mathbf{A}_{0}$, at least one agent $i$ has $x_{i} \in$ int $X_{i}$ and $\partial u_{i}\left(x_{i}\right)$ a singleton. Then $\mathbf{A}_{0}=\mathbf{E}$.

The following main result synthesizes the preceding facts:

Theorem 4.5 (Convergence to equilibrium). Under the conditions in Theorem 4.4 suppose that the hypotheses of Propositions 4.1 or 4.2 hold. Also suppose the set of feasible allocations is bounded. Then, the exchange process accumulates, and each limit point is an equilibrium profile supported by a market clearing price vector.

\footnotetext{
${ }^{9}$ Examples include $\mathrm{CO}_{2}$-equivalents (as regulated by the Kyoto agreement). Otherwise, when resource supply is at stake, water is a case in point.
} 


\section{Differences in Gradients and Margins}

Real transfers (11) was motivated in terms of the steepest slope - a rather geometric concept. What is the underlying economic and practical significance? And how do convex data affect such matters? These are the issues addressed in this somewhat technical section. It can be skipped with little loss of economic substance.

I now fix attention on two agents $i, j$, actually holding $\left(x_{i}, x_{j}\right) \in X_{i} \times X_{j}$. Throughout this section, suppose data are convex in the sense that all objects $-u_{i},-u_{j}, X_{i}$, $X_{j}$ are convex.

For motivation, suppose the agents see a well-defined gradient difference $\Delta:=$ $u_{i}^{\prime}\left(x_{i}\right)-u_{j}^{\prime}\left(x_{j}\right) \neq 0$ at $\left(x_{i}, x_{j}\right) \in \operatorname{int}\left(X_{i} \times X_{j}\right)$. The normalized difference $d:=\Delta /\|\Delta\|$ then becomes a best direction, giving steepest slope

$$
\mathfrak{S}_{i j}\left(x_{i}, x_{j}, d\right)=\left\|u_{i}^{\prime}\left(x_{i}\right)-u_{j}^{\prime}\left(x_{j}\right)\right\|=\mathfrak{S}_{i j}\left(x_{i}, x_{j}\right) .
$$

How are these simple facts modified if the gradient difference isn't well-defined or if $\left(x_{i}, x_{j}\right) \notin \operatorname{int}\left(X_{i} \times X_{j}\right)$ ? To spell out how, I recall (2) to say that agents $i, j$ see $a$ common price vector iff

$$
\left[\partial u_{i}\left(x_{i}\right)-N_{i}\left(x_{i}\right)\right] \cap\left[\partial u_{j}\left(x_{j}\right)-N_{j}\left(x_{j}\right)\right] \neq \emptyset .
$$

By tacit assumption, the concerned parties need no time to decide whether (15) holds or not. Since $0 \in N_{i}\left(x_{i}\right) \cap N_{j}\left(x_{j}\right)$, it suffices for (15) that $\partial u_{i}\left(x_{i}\right) \cap \partial u_{j}\left(x_{j}\right)$ be nonempty. When moreover, $u_{i}, u_{j}$ are differentiable, this simply means $u_{i}^{\prime}\left(x_{i}\right)=u_{j}^{\prime}\left(x_{j}\right)$.

Note that problem (5) amounts to sup-convolution (1) when $I=\{i, j\}$ and $x=x_{i}+x_{j}$ This observation invites another take on existence of common prices:

Proposition 5.1 (A common price blocks bilateral reallocation). For convex data, suppose there exists $\left(\tilde{x}_{i}, \tilde{x}_{j}\right) \in \operatorname{int}\left(X_{i} \times X_{j}\right)$ such that $\tilde{x}_{i}+\tilde{x}_{j}=x_{i}+x_{j}$. Then, at any optimal solution $\left(x_{i}^{+1}, x_{j}^{+1}\right)$ to problem (5), agents $i, j$ see a common price vector (15).

Conversely, if indeed they see one such vector at $\left(x_{i}^{+1}, x_{j}^{+1}\right) \in X_{i} \times X_{j}$, and $x_{i}^{+1}+x_{j}^{+1}=x_{i}+x_{j}$, then $\left(x_{i}^{+1}, x_{j}^{+1}\right)$ solves $(5)$.

Proposition 5.1, while serving as a reference, gives priority to duality and prices. It says that two agents who see a common price vector, shouldn't contemplate any bilateral barter; they better walk separate ways. Put differently: when their margins (and substitution rates) coincide, they have no incentives to make a deal.

A simplified version of Proposition 2.2 repeats the same message, but in primal terms:

Proposition 5.2 (At bilateral optimum no common direction offers improvement). For convex data, the pair $\left(x_{i}, x_{j}\right)$ solves problem (5) iff

$$
u_{i}^{\prime}\left(x_{i} ; d\right)+u_{j}^{\prime}\left(x_{j} ;-d\right) \leq 0 \text { for each } d \in D_{i j}\left(x_{i}, x_{j}\right) .
$$


Taken together, Propositions 5.1\&2 invite the following question: Suppose agents $i, j$ see no common price vector albeit a common feasible direction; that is, suppose

$$
\left[\partial u_{i}\left(x_{i}\right)-N_{i}\left(x_{i}\right)\right] \cap\left[\partial u_{j}\left(x_{j}\right)-N_{j}\left(x_{j}\right)\right]=\varnothing \text { yet } D_{i j}\left(x_{i}, x_{j}\right) \neq\{0\} .
$$

On such an occasion, how is the steepest slope characterized? To see how, let $P_{i j}[x]:=t$ denote the projection of $x \in \mathbb{X}$ onto the closure $\bar{D}_{i j}\left(x_{i}, x_{j}\right)$ of $D_{i j}\left(x_{i}, x_{j}\right){ }^{10}$ As one expects, such projection informs about the prospects for joint improvement:

Proposition 5.3 (The steepest slope as a projected gradient difference). For convex data, the steepest slope equals

$$
\begin{aligned}
\mathfrak{S}_{i j}\left(x_{i}, x_{j}\right) & =\max \left\{u_{i}^{\prime}\left(x_{i} ; d\right)+u_{j}^{\prime}\left(x_{j} ;-d\right) \mid d \in \bar{D}_{i j}\left(x_{i}, x_{j}\right) \&\|d\| \leq 1\right\} \\
& =\min \left\{\left\|P_{i j}\left[g_{i}-g_{j}\right]\right\| \mid g_{i} \in \partial u_{i}\left(x_{i}\right), g_{j} \in \partial u_{j}\left(x_{j}\right)\right\} .
\end{aligned}
$$

In the convenient case, when $\left(x_{i}, x_{j}\right) \in \operatorname{int}\left(X_{i} \times X_{j}\right)$,

$$
\mathfrak{S}_{i j}\left(x_{i}, x_{j}\right)=\min \left\{\left\|g_{i}-g_{j}\right\| \mid g_{i} \in \partial u_{i}\left(x_{i}\right), g_{j} \in \partial u_{j}\left(x_{j}\right)\right\} .
$$

If moreover, $u_{i}$ and $u_{j}$ are differentiable at $x_{i}$ and $x_{j}$ respectively, (14) holds.

The last observations indicate the possibly expedient roles played by agents with smooth objectives and interior choice. In particular, it appears that presence of barrier-like criteria - e.g. where $X_{i}$ is an orthant, and $u_{i}$ a Cobb-Douglas or logarithmic type function - can simplify some transactions, and make many others superfluous; confer the main Theorem 4.5. In short, it facilitates exchange that everybody trade regularly with some "smooth, interior-point" agent.

Lower semicontinuity was crucial in Propositions 4.1\&2. The projected gradient difference in Proposition 5.3 helps to argue that such continuity is natural. For the statement, given any two sets $\mathcal{C}_{i}, \mathcal{C}_{j} \subset \mathbb{X}$, their distance is

$$
\operatorname{dist}\left(\mathcal{C}_{i}, \mathcal{C}_{j}\right):=\inf \left\|\mathcal{C}_{i}-\mathcal{C}_{j}\right\|=\inf \left\{\left\|c_{i}-c_{j}\right\| \mid c_{i} \in \mathcal{C}_{i}, c_{j} \in \mathcal{C}_{j}\right\}
$$

Proposition 5.4 (Lower semicontinuity of the steepest slope). For convex data,

$$
\begin{aligned}
\mathfrak{S}_{i j}\left(x_{i}, x_{j}\right) & =\operatorname{dist}\left[\partial u_{i}\left(x_{i}\right)-N_{i}\left(x_{i}\right), \partial u_{j}\left(x_{j}\right)-N_{j}\left(x_{j}\right)\right] \\
& =\operatorname{dist}\left[0, \partial u_{i}\left(x_{i}\right)-N_{i}\left(x_{i}\right)-\partial u_{j}\left(x_{j}\right)+N_{j}\left(x_{j}\right)\right] .
\end{aligned}
$$

It follows that $\mathfrak{S}_{i j}$ is lower semicontinuous on $X_{i} \times X_{j}$. Further, $\mathfrak{S}_{i j}\left(x_{i}, x_{j}\right)=0$ iff agents $i, j$ see a common price (15). Hence $\left(x_{i}, x_{j}\right)$ already solves problem (5) if

$$
0 \in P_{i j}\left[\partial u_{i}\left(x_{i}\right)-\partial u_{j}\left(x_{j}\right)\right]
$$

\footnotetext{
${ }^{10}$ I ought write $P_{i j}\left[\cdot, x_{i}, x_{j}\right]$ for the operator $P_{i j}[\cdot]$. The pair $\left(x_{i}, x_{j}\right)$ is, however, tacitly understood or clear from the context.
} 
When at some feasible allocation $\mathbf{x}=\left(x_{i}\right)$ all $\mathfrak{S}_{i j}\left(x_{i}, x_{j}\right)=0$, every agent pair detects a common price. Accordingly, by Proposition 5.4, if the process arrives at such a point, trade stops. ${ }^{11}$

Concluding this section is an upper bound on the payoff increment (8) - to be followed by a remark on assumption (13).

Proposition 5.5 (Overestimating the payoff increment). Given convex data, it holds for any unit direction $d \in D_{i j}\left(x_{i}, x_{j}\right)$ and sufficiently small step-size $\sigma>0$ that

$$
\Delta u_{i j}(\sigma) \leq \sigma \mathfrak{S}_{i j}\left(x_{i}, x_{j}\right) .
$$

Consequently, under real transfers,

$$
\Delta u_{i j}(\sigma) \rightarrow 0 \quad \text { iff } \quad \sigma \mathfrak{S}_{i j}\left(x_{i}, x_{j}\right) \rightarrow 0
$$

A remark on assumption (13). Admittedly, the justification given for (13) might appear somewhat ad hoc or simply geared towards sufficiency. Yet, (13) appears necessary as indicated next.

Consider an economy which comprises only two agents $i, j$ who make real transfers, have $u_{i}, u_{j}$ concave, and $X_{i}, X_{j}$ bounded so that supergradients of $u_{i}, u_{j}$ are normbounded by some $\beta>0$. Thus, for any feasible allocation $\left(x_{i}, x_{j}\right)$ and $g_{i} \in \partial u_{i}\left(x_{i}\right)$, $g_{j} \in \partial u_{j}\left(x_{j}\right)$ it follows from Proposition 5.3 that

$$
\mathfrak{S}_{i j}\left(x_{i}, x_{j}\right) \leq\left\|P_{i j}\left(g_{i}-g_{j}\right)\right\| \leq\left\|g_{i}-g_{j}\right\| \leq 2 \max \left\{\left\|g_{i}\right\|,\left\|g_{j}\right\|\right\} \leq 2 \beta .
$$

Proposition 5.5 implies

$$
\sum_{k=0}^{\infty} \Delta u_{i j}^{k} \leq 2 \beta \sum_{k=0}^{\infty} \sigma^{k} .
$$

Consequently, the two parties would never reach an efficient outcome if

$$
2 \beta \sum_{k=0}^{\infty} \sigma^{k}<\max \left\{U(\mathbf{x})-U\left(\mathbf{x}^{0}\right) \mid \mathbf{x} \text { feasible }\right\} .
$$

\section{Three EXAMPles}

This section illustrates the exchange process, first by a simple numerical example. Thereafter it brings out two important, fairly general instances. One concerns linear production games; the other deals with trade of contingent claims under asymmetric information.

\footnotetext{
${ }^{11}$ By Propositions 5.1-3, agents $i, j$ shouldn't barter if

* they see a common price (15), or

* they detect no proper common direction: $D_{i j}\left(x_{i}, x_{j}\right)=\{0\}$, or

* their difference in margins is orthogonal to such directions: $0 \in P_{i j}\left[\partial u_{i}\left(x_{i}\right)-\partial u_{j}\left(x_{j}\right)\right]$. In each case, $\mathfrak{S}_{i j}\left(x_{i}, x_{j}\right)=0$.
} 
In all examples the cone $D_{i}\left(x_{i}\right)$ of feasible directions is closed convex. Also, the best direction is easily found.

Example 6.1. (Exchange when some margins are nil). Following Goldman and Starr [20] consider agents $i=1,2,3$, troubled by absence of double coincidence of wants. Let $S=\{1,2,3\}$; use $\mathbb{X}=\mathbb{R}^{S}$ with standard inner product; choose $X_{i}=\mathbb{R}_{+}^{S}$, and posit linear objectives

$$
\begin{array}{ll}
u_{1}\left(x_{1}\right)=2 x_{11}+1 x_{12}+0 x_{13}, & e_{1}=(1,1,0), \\
u_{2}\left(x_{2}\right)=0 x_{21}+2 x_{22}+1 x_{23}, & e_{2}=(0,1,1), \\
u_{3}\left(x_{3}\right)=1 x_{31}+0 x_{32}+2 x_{33}, & e_{3}=(1,0,1)
\end{array}
$$

Let agent pairs $\{1,2\},\{1,3\},\{2,3\}$ trade in that order. Start from $\left[x_{i}^{0}\right]=\left[e_{i}\right]$, invariably use the best direction $d=P_{i j}\left[u_{i}^{\prime}-u_{j}^{\prime}\right]$ and step-size $\sigma=1$, to get

$$
\begin{array}{lll}
\text { first: } & d=P_{12}\left[u_{1}^{\prime}-u_{2}^{\prime}\right]=(0,-1,0) \Rightarrow\left[x_{i}^{1}\right]=[(1,0,0),(0,2,1),(1,0,1)], \\
\text { second: } & d=P_{13}\left[u_{1}^{\prime}-u_{3}^{\prime}\right]=(1,0,0) \Rightarrow\left[x_{i}^{2}\right]=[(2,0,0),(0,2,1),(0,0,1)], \\
\text { third: } & d=P_{23}\left[u_{2}^{\prime}-u_{3}^{\prime}\right]=(0,0,-1) \Rightarrow\left[x_{i}^{3}\right]=[(2,0,0),(0,2,0),(0,0,2)],
\end{array}
$$

and thus finally, the efficient allocation. The equilibrium price $p=(2,2,2)$ becomes common (2) by choosing normals $\left[n_{i}\right]=-[(0,1,0),(0,0,1),(1,0,2)] \in \Pi_{i} N_{i}\left(x_{i}\right)$.

In each round, the two traders gain $\Delta u_{i j}=1$. The receiving agent gets additional payoff 2, and the donor gives up 1. So, if the first compensates the other with 1.5, both gain 0.5 .

The preceding instance invites a brief glance at an important class of economies that feature both exchange and production:

Example 6.2: Linear production games. Let

$$
u_{i}\left(x_{i}\right):=\sup \left\{y_{i}^{*} \cdot y \mid x_{i} \geq A_{i} y_{i} \& y_{i} \geq 0\right\} .
$$

Here the "price-vector" $y_{i}^{*}$ and the "activity plan" $y_{i}$ both belong to a Euclidean space $\mathbb{Y}_{i}$. That space and $\mathbb{X}$ are equipped with standard vector orders and inner products. The linear mapping $A_{i}: \mathbb{Y}_{i} \rightarrow \mathbb{X}$ represents a technology that consumes various production factors - of which the bundle $x_{i}$ is available. ${ }^{12}$

Conveniently, by Danskin's envelope theorem, $x_{i}^{*} \in \partial u_{i}\left(x_{i}\right)$ iff $x_{i}^{*}$ solves the dual to problem (16), namely:

$$
\inf \left\{x_{i}^{*} \cdot x_{i} \mid A_{i}^{T} x_{i}^{*} \geq y_{i}^{*} \& x_{i}^{*} \geq 0\right\} .
$$

Moreover, the cone $D_{i}\left(x_{i}\right)$ is closed convex and easily computable at any feasible $x_{i}$. Indeed, if $\mathbb{X}=\mathbb{R}^{S}$ for some finite list $S$ of goods, the index ensemble

$$
S_{i}\left(x_{i}\right):=\left\{s \in S \mid\left[x_{i}-A_{i} y_{i}\right]_{s}=0 \text { and } y_{i} \text { is optimal in }(16)\right\}
$$

\footnotetext{
${ }^{12}$ Agent $i^{\prime}$ s feasible set $X_{i}:=u_{i}^{-1}(\mathbb{R})$.
} 
identifies the binding constraints. Then $D_{i}\left(x_{i}\right)$ equals the "orthant" that has $\mathbb{R}_{+}$in each component $s \in S_{i}\left(x_{i}\right)$, and the entire line $\mathbb{R}$ in all others. Hence projection onto $D_{i j}\left(x_{i}, x_{j}\right)$ is easily executed.

In principle, extensions to non-linear production games are immediate. Specifically, in analogy with (16), let the reduced utility

$$
u_{i}\left(x_{i}\right):=\sup \left\{f_{i}\left(y_{i}\right) \mid x_{i} \geq g_{i}\left(y_{i}\right), y_{i} \in Y_{i}\right\}
$$

stem from convex functions $-f_{i}: \mathbb{Y}_{i} \rightarrow \mathbb{R}, g_{i}: \mathbb{Y}_{i} \rightarrow \mathbb{X}$, and a closed convex set $Y_{i} \subseteq \mathbb{Y}$. Then, to find supergradients $x_{i}^{*} \in \partial u_{i}\left(x_{i}\right)$ - alias Lagrange multipliers - is usually harder. But again, to identify $D_{i}\left(x_{i}\right)$ amounts only to assess which constraints are binding.

Example 6.3: Contingent claims and asymmetric information ex post [11]. In the setting of finance or insurance, plagued by uncertainty about the future, let $S$ denote a finite, full set of relevant, but mutually exclusive states $s \in S$. Posit $\mathbb{X}=\mathbb{R}^{S}$ as the linear space of all contingent claims $x: S \rightarrow \mathbb{R}$ to money.

Such a claim $x \in \mathbb{X}$ is adapted to a partition $\mathcal{P}$ of $S$, written $x \in \mathcal{A}(\mathcal{P})$, if $x$ is constant on each part $P \in \mathcal{P}$. Agent $i$ is information constrained iff $X_{i} \subseteq \mathcal{A}\left(\mathcal{P}_{i}\right)$ for some proper partition $\mathcal{P}_{i}$ of $S$. In particular, when $X_{i}=\mathcal{A}\left(\mathcal{P}_{i}\right)$, agent $i$ can identify state $s$ ex post only up to the part $P(s) \in \mathcal{P}_{i}$ which contains $s$. Agents $i, j$ have asymmetric information structures if $\mathcal{P}_{i} \neq \mathcal{P}_{j}$; see [11].

Of particular importance are instances $X_{i}=\mathcal{A}\left(\mathcal{P}_{i}\right)$ for each $i \in I$. Then, since $\mathcal{A}\left(\mathcal{P}_{i}\right)$ is a closed linear subspace, each direction $d \in D_{i j}\left(x_{i}, x_{j}\right)=X_{i} \cap X_{j}$ must be constant on $P_{i} \cup P_{j}$ whenever the parts $P_{i} \in \mathcal{P}_{i}$ and $P_{j} \in \mathcal{P}_{j}$ intersect.

Many agent pairs $i, j$ may see few feasible barters of mutual interest. In contrast, suppose a particular party $j$ enjoys the most fine-grained information, his state-space partition $\mathcal{P}_{j}$ being composed only of singletons. Since $X_{j}=\mathbb{X}$, presence of such a well-informed "intermediary" or broker $j$ largely facilitates trade.

As to computation, suppose scenario $s \in S$ comes up with objective probability $\pi_{s}>0, \sum_{s \in S} \pi_{s}=1$. Endow $\mathbb{X}$ with probabilistic inner product $\left\langle x^{*}, x\right\rangle:=$ $\sum_{s \in S} x_{s}^{*} x_{s} \pi_{s}$ and corresponding norm. Then, on part $P$ of a partition $\mathcal{P}$ of $S$, the projection $\operatorname{Pr}(x)$ of $x \in \mathbb{X}$ onto the subset $X:=\mathcal{A}(\mathcal{P})$ equals the conditional expectation

$$
\operatorname{Pr}(x)_{s}=\frac{\sum_{s \in P} x_{s} \pi_{s}}{\sum_{s \in P} \pi_{s}} \text { for each } s \in P . \diamond
$$

\section{Concluding Remarks}

When must agent-based, decentralized choices be coordinated somehow? That intricate question is common to diverse fields, including computation, economics, and political science.

This paper has considered a particularly important case - namely, endowment exchange - and found it somewhat singular: under weak conditions, no coordination is needed. Even quite simple modes of market operation may suffice for convergence. 
In fact, a market isn't always needed. It can suffice that bilateral barters be the only vehicle.

Admittedly, while emphasizing mathematical aspects, the above narrative was scarce on institutional detail and description. Further, it considered merely two traders at a time - and played down their anonymity. The opposite, traditional optic - in which economic agents are linked only through perfect, fully anonymous markets - is well studied, but not always convincing; see Kirman (2010). In particular, it does not quite fit emerging markets.

I have, with no excuse, accommodated agents of bounded rationality, each with a local, limited perspective. Chief concerns were with market stability. The rate of convergence remains a secondary issue. No claims were made in that regard, and stages were not related to real time. It deserves emphasis, however, that most markets display a permanent bid-ask spread no less than some monetary tick $\varepsilon>0$. So, for practical purposes, what replaces the commonality of prices (2) is

$$
\cap_{i \in I}\left[\partial u_{i}\left(x_{i}\right)-N_{i}\left(x_{i}\right)+\varepsilon B\right] \neq \varnothing,
$$

$B$ being the closed unit ball. Then any $p$ from the above intersection qualifies as approximate equilibrium price. Upon allowing such "fuzzy" prices, convergence may prove fairly rapid - possibly finite.

One important obstacle has not been mentioned though, namely: The market topology might prevent that some agents meet; only conveniently connected "neighbors" can interact; see [21], [30]. ${ }^{13}$ Attainment of fully integrated market equilibrium then becomes more problematic. In extremis, the economy might decompose into disconnected autarkies. However, presence of well-connected middlemen - who preferably choose interior bundles and have smooth objectives - will enhance efficiency, and, at best, bring about convergence [39]. It appears worthwhile to explore how bilateral exchange, as modelled above, will fare in such settings.

I have also ignored that some constraints could be implicit. To wit, only finitevalued functions $u_{i}$ were admitted above. Instances like Example 6.2 may, however, feature effective domains domu $u_{i}:=u_{i}^{-1}(\mathbb{R})$ that are proper subsets of $\mathbb{X}$. When $X_{i}$ is interior to $d o m u_{i}$, no problems emerge. Otherwise, agent $i$ must care that $x_{i} \in X_{i} \cap d_{o m} u_{i}$. Such concerns motivate further studies.

To conclude, it is appropriate to mention that barters and commodity transfers, by themselves, may generate extra cost or cause some inertia. Yet here, they were implemented with no expense or hesitation. To mitigate this objectionable feature, one could envisage that agent $i$ invokes a regularized objective

$$
u_{i}(x):=\max \left\{\tilde{u}_{i}(\tilde{x})-a_{i}(\tilde{x}, x) \mid \tilde{x} \in \mathbb{X}\right\} .
$$

The underlying $\tilde{u}_{i}: \mathbb{X} \rightarrow \mathbb{R} \cup\{-\infty\}$ reports his proper revenue whereas $a_{i}: \mathbb{X} \times \mathbb{X} \rightarrow \mathbb{R}$ accounts for adjustment or transaction cost. Presumably, $a_{i}(\tilde{x}, x) \geq 0$, and $a_{i}(x, x)=$ 0 . Quite reasonably, the regularization could also require that $\tilde{x} \in X_{i}$.

\footnotetext{
${ }^{13}$ Numerous papers study such settings; see e.g. [6], [22], [29].
} 
Besides its appeal, that operation often brings an extra bonus: it is apt to smoothen the resulting objective. Specifically, provided $\tilde{u}_{i}(\tilde{x})-a_{i}(\tilde{x}, x)$ be strictly concave in $\tilde{x}$ and differentiable in $x$, the maximizing $\tilde{x}=\tilde{x}(x)$ is unique, and - by Danskin's envelope theorem [7] - the derived criterion $u_{i}$ becomes differentiable with

$$
u_{i}^{\prime}(x)=-\frac{\partial}{\partial x} a_{i}(\tilde{x}, x) .
$$

Plainly, an agent who regularizes his objective, appears competent as optimizer. But that feature does not square with how he was portrayed. So, although interesting, I have not considered the effects of iterated regularizations. 


\section{Appendix: Proofs}

Proof of Theorem 2.1. In essence, the first bullet was already proved in [12]. For completeness the demonstration is included. Define the "death" penalty $\delta_{0}(\cdot)$ on $\mathbb{X}$ by $\delta_{0}(\chi)=-\infty$ when $\chi \neq 0$ and $\delta_{0}(0)=0$. Note that $\sup _{\chi}\left\{\delta_{0}(\chi)-\left\langle x^{*}, \chi\right\rangle\right\}=0$ for any $x^{*} \in \mathbb{X}$. Now, $p \in \partial u_{I}\left(e_{I}\right)$

$$
\begin{aligned}
& \Leftrightarrow \sum_{i \in I} \check{u}_{i}\left(\chi_{i}\right)+\delta_{0}\left(\chi-\sum_{i \in I} \chi_{i}\right) \leq u_{I}(\chi) \leq u_{I}\left(e_{I}\right)+\left\langle p, \chi-e_{I}\right\rangle \forall \chi \in \mathbb{X},\left(\chi_{i}\right) \in \mathbb{X}^{I} \\
& \Leftrightarrow \sum_{i \in I} \check{u}_{i}\left(\chi_{i}\right)+\sum_{i \in I}\left\langle p, e_{i}-\chi_{i}\right\rangle+\delta_{0}\left(\chi-\sum_{i \in I} \chi_{i}\right)-\left\langle p, \chi-\sum_{i \in I} \chi_{i}\right\rangle \leq u_{I}\left(e_{I}\right) \forall \chi,\left(\chi_{i}\right) \\
& \Leftrightarrow \sum_{i \in I}\left\{\check{u}_{i}\left(\chi_{i}\right)+\left\langle p, e_{i}-\chi_{i}\right\rangle\right\} \leq u_{I}\left(e_{I}\right) \quad \forall\left(\chi_{i}\right) \in \mathbb{X}^{I} .
\end{aligned}
$$

Let $\left(x_{i}\right)$ be any efficient allocation. Then the above string of equivalences says that $p \in \partial u_{I}\left(e_{I}\right)$ iff

$$
\sum_{i \in I} \check{u}_{i}\left(\chi_{i}\right) \leq \sum_{i \in I}\left\{\check{u}_{i}\left(x_{i}\right)+\left\langle p, \chi_{i}-x_{i}\right\rangle\right\} \forall\left(\chi_{i}\right) \in \mathbb{X}^{I}
$$

Consider any $i \in I$. In (17) posit $\chi_{j}=x_{j}$ when $j \neq i$ to have

$$
\breve{u}_{i}\left(\chi_{i}\right) \leq \breve{u}_{i}\left(x_{i}\right)+\left\langle p, \chi_{i}-x_{i}\right\rangle \text { for all } \chi_{i} \in \mathbb{X} \text {, and } i \in I \text {. }
$$

So, $p \in \partial \check{u}_{i}\left(x_{i}\right), \forall i$. Conversely, summing the last inequalities over $i$ yields (17). This proves the first bullet on common margins.

For the second bullet, simply note that the very last system of inequalities amounts to the equilibrium conditions:

$$
u_{i}\left(\chi_{i}\right)+\left\langle p, e_{i}-\chi_{i}\right\rangle \leq u_{i}\left(x_{i}\right)+\left\langle p, e_{i}-x_{i}\right\rangle \text { for all } \chi_{i} \in X_{i} \text {, and } i \in I .
$$

Moreover, when $\left(\chi_{i}\right)$ is a feasible allocation, summation of the said conditions gives $\sum_{i \in I} u_{i}\left(\chi_{i}\right) \leq \sum_{i \in I} u_{i}\left(x_{i}\right)$. That is, any equilibrium profile is efficient. Further, by repeating the same summation, but now with $\sum_{i \in I} \chi_{i}=\chi$, we obtain

$$
u_{I}(\chi) \leq u_{I}\left(e_{I}\right)+\left\langle p, \chi-e_{I}\right\rangle \text { for all } \chi,
$$

hence $p \in \partial u_{I}\left(e_{I}\right)$.

For the third bullet, suppose an allocation $\left(x_{i}\right)$ does not belong to the compact set $\prod_{i \in \mathcal{I}} X_{i} \times \prod_{i \notin \mathcal{I}} r B$ where $B$ denotes the closed unit ball in $\mathbb{X}$. Then, for $r$ large enough, $\sum_{i \in I} u_{i}\left(x_{i}\right)<u_{I}\left(e_{I}\right)$. Hence existence of an efficient allocation derives from the upper semicontinuity of each $u_{i}$.

Finally, for existence of a shadow price, observe that $\partial \hat{u}_{I}\left(e_{I}\right) \subseteq \partial u_{I}\left(e_{I}\right)$. 
Proof of Corollary 2.1. For any subset $X \subseteq \mathbb{X}$ let the extended indicator $\delta_{X}$ equal 0 on $X$, and $-\infty$ elsewhere. Then $N_{X}(x)=-\partial \delta_{X}(x)$. Suppose (2) holds for some allocation $\left(x_{i}\right)$. Since $N_{i}$ is empty outside $X_{i}$, we must have $x_{i} \in X_{i}$ - to the effect that the allocation at hand is feasible. Further, $p \in \partial u_{i}\left(x_{i}\right)-N_{i}\left(x_{i}\right)$ amounts to

$$
0 \in \partial\left[u_{i}+\left\langle p, e_{i}-\cdot\right\rangle+\delta_{X_{i}}\right]\left(x_{i}\right),
$$

which is the optimality condition for an agent $i$ who faces price vector $p$. Conversely, when $(\mathbf{x}, p)$ is an equilibrium, by Theorem 2.1, $p \in \partial \check{u}_{i}\left(x_{i}\right)=\partial u_{i}\left(x_{i}\right)-N_{i}\left(x_{i}\right)$.

Proof of Proposition 2.2. Provided $\sum_{i \in I}\left(x_{i}, d_{i}\right)=\left(e_{I}, 0\right)$, it holds that $\sum_{i \in I}\left(x_{i}+\right.$ $\left.r d_{i}\right)=e_{I}$ for any real $r$. Then, with $d_{i} \in D_{i}\left(x_{i}\right)$ for each $i$, and $\left(x_{i}\right)$ efficient, $\sum_{i \in I}\left[u_{i}\left(x_{i}+r d_{i}\right)-u_{i}\left(x_{i}\right)\right] \leq 0$ for small enough $r>0$, whence $\sum_{i \in I} u_{i}^{-}\left(x_{i} ; d_{i}\right) \leq 0$.

Conversely, suppose $\left(x_{i}\right)$ be strictly dominated by another feasible allocation $\left(\chi_{i}\right)$. Posit $d_{i}:=\chi_{i}-x_{i}$ to have $\sum_{i \in I} d_{i}=0$. Clearly, when $X_{i}$ is convex, $d_{i} \in D_{i}\left(x_{i}\right)$. Further, presuming $u_{i}$ concave,

$$
u_{i}^{-}\left(x_{i} ; d_{i}\right) \geq \frac{u_{i}\left(x_{i}+r d_{i}\right)-u_{i}\left(x_{i}\right)}{r} \text { for each } r>0
$$

so that with $r=1$,

$$
\sum_{i \in I} u_{i}^{-}\left(x_{i} ; d_{i}\right) \geq \sum_{i \in I}\left[u_{i}\left(x_{i}+d_{i}\right)-u_{i}\left(x_{i}\right)\right]=\sum_{i \in I}\left[u_{i}\left(\chi_{i}\right)-u_{i}\left(x_{i}\right)\right]>0 .
$$

Proof of Proposition 3.1. By definition (10) there exists $d \in D_{i j}\left(x_{i}, x_{j}\right),\|d\| \leq 1$, such that

$$
\mathfrak{S}_{i j}\left(x_{i}, x_{j}, d\right) \geq \varphi_{i j}^{1 / 2} \mathfrak{S}_{i j}\left(x_{i}, x_{j}\right) .
$$

The positive homogeneity of the directional derivatives ensures that we may take $\|d\|=1$. For small enough $\sigma>0$, inclusions (7) hold and

$$
\Delta u_{i j}\left(x_{i}, x_{j}, \sigma, d\right) \geq \sigma \varphi_{i j}^{1 / 2} \mathfrak{S}_{i j}\left(x_{i}, x_{j}, d\right) .
$$

Combining the last two inequalities, (11) follows forthwith.

Proof of Proposition 4.1. Consider the aggregate payoff function

$$
U(\mathbf{x}):=\sum_{i \in I} u_{i}\left(x_{i}\right)
$$

Real transfers (11) imply that $U\left(\mathbf{x}^{k}\right)=\sum_{i \in I} u_{i}\left(x_{i}^{k}\right)$ steadily increases. Since $U\left(\mathbf{x}^{k}\right) \leq$ $u_{I}\left(e_{I}\right)<+\infty, \lim _{k \rightarrow \infty} U\left(\mathbf{x}^{k}\right)$ is well defined and finite. Consequently,

$$
\lim _{k \rightarrow+\infty} \sigma^{k} \mathfrak{S}_{i j}\left(x_{i}^{k}, x_{j}^{k}\right)=0 \text { for each agent pair } i, j .
$$


Otherwise (11) yields $\lim _{k \rightarrow \infty} U\left(\mathbf{x}^{k}\right)=+\infty$. Further, the entire sequence $\mathbf{x}^{k}=\left(x_{i}^{k}\right)$, $k=0,1, \ldots$ is asymptotically regular, meaning

$$
\left\|\mathbf{x}^{k+1}-\mathbf{x}^{k}\right\| \rightarrow 0
$$

(19) follows from $\sigma^{k} \rightarrow 0$ and

$$
\left\|\mathbf{x}^{k+1}-\mathbf{x}^{k}\right\|=\sigma^{k} 2^{1 / 2} .
$$

For the last equality use format (7) and $\|d\|=1$.

Let $\mathcal{L}$ denote the set of all accumulation points of the generated sequence $\mathbf{x}^{k}=$ $\left(x_{i}^{k}\right), k=0,1, \ldots$. Suppose $\mathcal{L}$ is non-empty and pick any $\mathbf{x} \in \mathcal{L}$. Then $\mathbf{x}=\lim _{k \in K} \mathbf{x}^{k}$ for some subsequence $K \subseteq\{0,1, \ldots\}$. If necessary, pass to a subsequence of $K$ in which the lapse between consecutive members is greater than $l$. Assume this is already done.

I claim that $\mathfrak{S}_{i j}\left(x_{i}, x_{j}\right)=0$ for every $i, j$. To arrive at a contradiction, suppose some agent pair $i, j$ has $\mathfrak{S}_{i j}\left(x_{i}, x_{j}\right)>0$. Let $\kappa(k)$ be the first stage $\geq k \in K$ at which $i, j$ trade. Thus emerges, by the quasi-cyclic nature of the encounters, a sequence $\mathcal{K}$ of stages $\kappa(k) \in[k, \ldots, k+l[, k \in K$, at which $i, j$ always trade. The asymptotic regularity (19) ensures that $\mathbf{x}=\lim _{\kappa \in \mathcal{K}} \mathbf{x}^{\kappa}$. By the lower semicontinuity of $\mathfrak{S}_{i j}$,

$$
\lim \inf _{\kappa \in \mathcal{K}} \mathfrak{S}_{i j}\left(x_{i}^{\kappa}, x_{j}^{\kappa}\right) \geq \mathfrak{S}_{i j}\left(x_{i}, x_{j}\right)>0 .
$$

(13) yields $\sum_{\kappa \in \mathcal{K}} \sigma_{i j}^{\kappa}=\infty$. Again (11) implies the contradiction $\lim _{k \rightarrow \infty} U\left(\mathbf{x}^{k}\right)=+\infty$. In conclusion, $\mathfrak{S}_{i j}\left(x_{i}, x_{j}\right)=0$ for each pair $i, j$.

Proof of Proposition 4.2. Let $\mathrm{A}$ denote the set of all feasible allocations. Its subset $\mathbf{A}_{0}:=\left\{\left(x_{i}\right) \in \mathbf{A} \mid\right.$ all $\left.\mathfrak{S}_{i j}\left(x_{i}, x_{j}\right)=0\right\}$ is of prime interest.

When $\mathbf{x} \in \mathbf{A}$, let $\mathbf{d}_{i j} \in \mathbb{X}^{I}$ have all components 0 except $i, j$ which feature unit vectors $d_{i}$ and $d_{j}$ respectively such that $d_{i}+d_{j}=0$.

Define $\varphi:=\min _{i j} \varphi_{i j}$. At each profile $\mathbf{x} \in \mathbf{A} \backslash \mathbf{A}_{0} \operatorname{posit} \mathfrak{S}(\mathbf{x}):=\max _{i, j} \mathfrak{S}_{i j}\left(x_{i}, x_{j}\right)$ and

$\mathbf{B}(\mathbf{x}):=\left\{\mathbf{x}^{+1}=\mathbf{x}+\sigma_{i j} \mathbf{d}_{i j} \in \mathbf{A} \mid \sigma_{i j} \geq \underline{\sigma}_{i j}\left(x_{i}, x_{j}\right)\right.$ and $\left.U\left(\mathbf{x}^{+1}\right) \geq U(\mathbf{x})+\sigma_{i j} \varphi \mathfrak{S}(\mathbf{x})\right\}$.

In contrast, when $\mathbf{x} \in \mathbf{A}_{0}$, let $\mathbf{B}(\mathbf{x})=\{\mathbf{x}\}$. This point-to-set correspondence $\mathbf{B}: \mathbf{A} \rightsquigarrow \mathbf{A}$ is closed outside $\mathbf{A}_{0}$. Moreover, if $\mathbf{x} \notin \mathbf{A}_{0}$ and $\mathbf{x}^{+1} \in \mathbf{B}(\mathbf{x})$, then $U\left(\mathbf{x}^{+1}\right)>U(\mathbf{x})$. The conclusion now follows from Theorem 7.3.4. in [5].

Proof of Proposition 4.3. Since $\partial u_{i}\left(x_{i}\right)-N_{i}\left(x_{i}\right)$ and $\partial u_{j}\left(x_{j}\right)-N_{j}\left(x_{j}\right)$ are closed convex sets, it follows from $\mathfrak{S}_{i j}\left(x_{i}, x_{j}\right)=0$ that each two of them intersect; see Proposition 5.4. Since moreover, the sets just mentioned are real intervals (maybe degenerate), $\cap_{i \in I}\left[\partial u_{i}\left(x_{i}\right)-N_{i}\left(x_{i}\right)\right]$ is non-empty. Proposition 2.1 tells that any $p$ in this intersection serves as an equilibrium price. 
Proof of Proposition 5.1. Let $x:=x_{i}+x_{j}$ and write

$$
\mathcal{B}(x):=\left\{\left(\hat{x}_{i}, \hat{x}_{j}\right) \in \mathbb{X} \times \mathbb{X} \mid \hat{x}_{i}+\hat{x}_{j}=x\right\}
$$

for the set of two-person balanced redistributions. Also for easier notation, given a constraint set $\mathcal{K}$, the extended lower penalty function $\delta_{\mathcal{K}}$ equals 0 on $\mathcal{K}$, and $-\infty$ elsewhere. Thus problem (5) assumes the equivalent form:

$$
\operatorname{maximize} u_{i}\left(x_{i}^{+1}\right)+\delta_{X_{i}}\left(x_{i}^{+1}\right)+u_{j}\left(x_{j}^{+1}\right)+\delta_{X_{j}}\left(x_{j}^{+1}\right)+\delta_{\mathcal{B}(x)}\left(x_{i}^{+1}, x_{j}^{+1}\right) .
$$

Because $\mathcal{B}(x) \cap \operatorname{int}\left(X_{i} \times X_{j}\right)$ is non-empty, the superdifferential operator $\partial$ distributes over the sum just stated. Further, since $\partial \delta_{X_{i}}=-N_{X_{i}}$, in optimum $\left(x_{i}^{+1}, x_{j}^{+1}\right)$ it holds

$$
[0,0] \in\left[\left(\partial u_{i}-N_{X_{i}}, \partial u_{j}-N_{X_{j}}\right)-N_{\mathcal{B}(x)}\right]\left(x_{i}^{+1}, x_{j}^{+1}\right) .
$$

$N_{\mathcal{B}(x)}\left(x_{i}^{+1}, x_{j}^{+1}\right)$ equals the "diagonal" $\left\{\left(x^{*}, x^{*}\right): x^{*} \in \mathbb{X}\right\}$ at each $\left(x_{i}^{+1}, x_{j}^{+1}\right) \in \mathcal{B}(x)$. So, it follows from the preceding inclusion of $[0,0]$ that there exists a common price

$$
x^{*} \in\left[\partial u_{i}\left(x_{i}^{+1}\right)-N_{X_{i}}\left(x_{i}^{+1}\right)\right] \cap\left[\partial u_{j}\left(x_{j}^{+1}\right)-N_{X_{j}}\left(x_{j}^{+1}\right)\right] .
$$

Conversely, given such a vector $x^{*}$, Corollary 2.1 tells that $\left(x_{i}^{+1}, x_{j}^{+1}\right)$ solves $(5)$.

Proof of Proposition 5.3. Recall that a concave function $f: \mathbb{X} \rightarrow \mathbb{R} \cup\{-\infty\}$ which is finite near $x \in \mathbb{X}$, has a non-empty compact convex superdifferential $\partial f(x)$ and a directional derivative

$$
f^{\prime}(x ; d):=\lim _{r \rightarrow 0^{+}} \frac{f(x+r d)-f(x)}{r}=\min \left\{\left\langle x^{*}, d\right\rangle: x^{*} \in \partial f(x)\right\} ;
$$

see Theorem 23.4 in [35]. Since $f^{\prime}(x ; d)$ is convex in $d$, it's continuous in that variable. This explains the first inequality. The second follows from $\mathfrak{S}_{i j}\left(x_{i}, x_{j}\right)=$

$$
\begin{aligned}
& \max _{d} \min _{g_{i}, g_{j}}\left\{\left\langle g_{i}-g_{j}, d\right\rangle \mid g_{i} \in \partial u_{i}\left(x_{i}\right), g_{j} \in \partial u_{j}\left(x_{j}\right), d \in \bar{D}_{i j}\left(x_{i}, x_{j}\right) \&\|d\| \leq 1\right\} \\
= & \min _{g_{i}, g_{j}} \max _{d}\left\{\left\langle g_{i}-g_{j}, d\right\rangle \mid g_{i} \in \partial u_{i}\left(x_{i}\right), g_{j} \in \partial u_{j}\left(x_{j}\right), d \in \bar{D}_{i j}\left(x_{i}, x_{j}\right) \&\|d\| \leq 1\right\} \\
= & \min \left\{\left\|P_{i j}\left[g_{i}-g_{j}\right]\right\| \mid g_{i} \in \partial u_{i}\left(x_{i}\right), g_{j} \in \partial u_{j}\left(x_{j}\right)\right\} .
\end{aligned}
$$

In the preceding string, the first equality used formula (21) and the definition of the steepest slope. Since all intervening sets are non-empty compact convex, the second equality follows from the von Neumann min-max theorem.

For the last equality, recall the following result of Moreau: Given a non-empty closed convex cone $T \subseteq \mathbb{X}$, any vector $\Delta \in \mathbb{X}$ has a unique orthogonal decomposition $\Delta=t+n$ into a "tangent" $t \in T$, and a normal $n \in N:=N_{T}(0)$, these components 
being perpendicular: $\langle t, n\rangle=0$; see Prop. 0.6.2 in [2]. Since $T=\bar{D}_{i j}\left(x_{i}, x_{j}\right)$ is indeed closed convex, let $\Delta=g_{i}-g_{j}$ and $t=P_{i j}[\Delta]$. Now the last equality in the above string derives from the Cauchy-Schwartz inequality, using the Moreau decomposition of the difference $\Delta$ with respect to $\bar{D}_{i j}\left(x_{i}, x_{j}\right)$.

For the proof of Proposition 5.4 we need a

Lemma (The distance between compact convex translates of closed convex cones). Suppose $\mathcal{C}_{i}=\mathcal{G}_{i}-\mathcal{N}_{i}, \mathcal{C}_{j}=\mathcal{G}_{j}-\mathcal{N}_{j}$, where $\mathcal{G}_{i}, \mathcal{G}_{j}$ are non-empty compact convex sets, and $\mathcal{N}_{i}, \mathcal{N}_{j}$ are closed convex cones. Define dual cones

$$
\mathcal{T}_{i}:=\left\{x^{*} \mid\left\langle x^{*}, \mathcal{N}_{i}\right\rangle \leq 0\right\}, \quad \mathcal{T}_{j}:=\left\{x^{*} \mid\left\langle x^{*}, \mathcal{N}_{j}\right\rangle \leq 0\right\}
$$

and posit $\mathcal{T}_{i j}:=\mathcal{T}_{i} \cap-\mathcal{T}_{j}$. Then, writing $P_{i j}$ for the orthogonal projection onto $\mathcal{T}_{i j}$, it holds

$$
\operatorname{dist}\left(\mathcal{C}_{i}, \mathcal{C}_{j}\right)=\min \left\{\left\|P_{i j}\left(g_{i}-g_{j}\right)\right\| \mid g_{i} \in \mathcal{G}_{i}, g_{j} \in \mathcal{G}_{j}\right\}=\left\|v_{i j}\right\| .
$$

The said distance is attained.

Proof of the Lemma. Bauschke and Borwein [3] have already considered the case where $\mathcal{G}_{i}, \mathcal{G}_{j}$ are singletons $g_{i}, g_{j}$ respectively. The more general result derives from $\operatorname{dist}\left(\mathcal{C}_{i}, \mathcal{C}_{j}\right)=$

$\inf \left\{\operatorname{dist}\left(g_{i}-\mathcal{N}_{i}, g_{j}-\mathcal{N}_{j}\right) \mid g_{i} \in \mathcal{G}_{i}, g_{j} \in \mathcal{G}_{j}\right\}=\min \left\{\left\|P_{i j}\left(g_{i}-g_{j}\right)\right\| \mid g_{i} \in \mathcal{G}_{i}, g_{j} \in \mathcal{G}_{j}\right\}$.

For the attainment of $\operatorname{dist}\left(\mathcal{C}_{i}, \mathcal{C}_{j}\right)$ simply note that $\mathcal{G}_{i}, \mathcal{G}_{j}$ are compact convex.

Proof of Proposition 5.4. Posit $\mathcal{G}_{i}:=\partial u_{i}\left(x_{i}\right), \mathcal{N}_{i}:=N_{i}\left(x_{i}\right)$, and similarly, $\mathcal{G}_{j}:=\partial u_{j}\left(x_{j}\right), \mathcal{N}_{j}:=N_{j}\left(x_{j}\right)$, to get $(22)$. The lower semicontinuity of $\mathfrak{S}_{i j}$ derives from the fact that all point-to-set correspondences in (22) are (upper) outer semicontinuous [36]. Further,

$$
\mathfrak{S}_{i j}\left(x_{i}, x_{j}\right)=\min \left\{\left\|P_{i j}\left(g_{i}-g_{j}\right)\right\| \mid g_{i} \in \mathcal{G}_{i}, g_{j} \in \mathcal{G}_{j}\right\}=\operatorname{dist}\left(\mathcal{C}_{i}, \mathcal{C}_{j}\right) .
$$

Here the left hand equality derives from Proposition 5.3 and the other from (22).

Proof of Proposition 5.5. Since both functions $u_{i}, u_{j}$ are concave, it holds for any supergradients $g_{i} \in \partial u_{i}\left(x_{i}\right), g_{j} \in \partial u_{j}\left(x_{i}\right)$, direction $d$, and step-size $\sigma$ that

$$
u_{i}\left(x_{i}^{+1}\right) \leq u_{i}\left(x_{i}\right)+\sigma\left\langle g_{i}, d\right\rangle \text { and } u_{j}\left(x_{j}^{+1}\right) \leq u_{j}\left(x_{j}\right)-\sigma\left\langle g_{j}, d\right\rangle .
$$

When moreover, $d \in D_{i j}\left(x_{i}, x_{j}\right)$, adding these two inequalities yields

$$
\Delta u_{i j} \leq \sigma\left\langle g_{i}-g_{j}, d\right\rangle \leq \sigma \mathfrak{S}_{i j}\left(x_{i}, x_{j}\right)\|d\|
$$




\section{REFERENCES}

[1] J. Andreoni and B. D. Bernheim, Social image and the 50-50 norm: a theoretical and experimental analysis of audience effects, Econometrica 77, 5, 1607-1636 (2009)

[2] J.P.Aubin and A. Cellina, Differential Inclusions, Springer Verlag, Berlin (1984)

[3] H. H. Bauschke and J. M. Borwein, On the convergence of von Neumann's alternating projection algorithm for two sets, Set-Valued Analysis 1, 183-212 (1993)

[4] H. H. Bauschke and J. M. Borwein, On projection algorithms for solving convex feasibility problems, SIAM Review 38, 3, 367-426 (1996)

[5] M. S. Bazaraa, H. D. Sherali, C.M. Shetty, Nonlinear Programming, J. Wiley \& Sons, New York (1993)

[6] M. Corominas-Bosch, Bargaining in a network of buyers and sellers, Journal of Economic Theory 155, 25-77 (2004)

[7] J. M. Danskin, The Theory of Max-Min and its Applications to Weapons Allocations Problems, Springer Verlag, Berlin (1967)

[8] E. Fehr and K. M. Schmidt, A theory of fairness, competition, and cooperation, The Quarterly Journal of Economics 817-868 (1999)

[9] A. M. Feldman, Bilateral trading processes, pairwise optimality, and Pareto optimality, The Review of Economic Studies 4, 463-473 (1973)

[10] F. M. Fisher, It takes t* to tango: Trading coalitions with fixed prices, The Review of Economic Studies 56, 391-404 (1989)

[11] S. D. Flåm and L. Koutsougeras, Private Information, Transferable Utility, and the Core, Economic Theory 42, 591-609 (2010)

[12] S. D. Flåm, On sharing of risks and resources, in S. Reich and A. Zaslavksi (eds.) Optimization Theory and Related Topics, American Mathematical Society, series Contemporary Mathematics 568, 53-68 (2012) http://dx.doi.org/10.1090/conm/568/11276

[13] S. D. Flåm, Exchanges and measures of risk, Mathematics and Financial Economics $5,4,249-267$ (2012)

[14] S. D. Flåm, O. Godal and A. Soubeyran, Gradient Differences and Bilateral Barters, Optimization 1-20, http://dx.doi.org/10.1080/02331934.2012.679940 (2012)

[15] S. D. Flåm, Coupled Projects, Core Imputations, and the CAPM, J. Mathematical Economics 48, 3, 170-176 (2012)

[16] S. D. Flåm and K. Gramstad, Direct exchanges in linear economies, to appear in Int. J. Game Theory (2013) 
[17] D. K. Gode and S. Sunder, Allocative efficiency of markets with zero intelligence traders: markets as a partial substitute for individual rationality, Journal of Political Economy 101, 119-37 (1993)

[18] S. Ghosal and M. Morelli, Retrading in markets, J. Economic Theory 115, 15-181 (2004)

[19] H. Gintis, The emergence of a price system from decentralized bilateral exchange, Contributions to Theoretical Economics 6,1, Art. 13 (2006)

[20] S. M. Goldman and R. M. Starr, Pairwise, t-wise, and Pareto optimalities, Econometrica 50, 3, 593-6066 (1982)

[21] M. O. Jackson, Social and Economic Networks, Princeton University Press (2008)

[22] R. E. Kranton and D. F. Minehart, A theory of buyer-seller networks, The American Economic Review 91, 3, 485-507 (2001)

[23] A. Kirman, The economic crisis is a crisis for economic theory, CESifo Economic Studies 56, 4, 498-535 (2010)

[24] D. Lucking-Reiley and D. F. Spulber, Business-to-Business electronic commerce, J. Economic Perspectives 15, 55-68 (2001)

[25] D. G. Luenberger, Linear and Nonlinear Programming, Addison-Wesley. London (1984)

[26] P. J. Madden, Efficient sequences of non-monetary exchange, The Review of Economic Studies 42, 4, 581-596 (1975)

[27] M. Magill and M. Quinzii, Theory of Incomplete Markets, MIT Press (1996)

[28] L. Makowski, A characterization of perfectly competitive economies with production, J. Economic Theory 22, 208-221 (1980)

[29] R. B. Myerson, Graphs and cooperation in games, Mathematics of Operations Research 2,3, 225-229 (1977)

[30] M. Mesbahi and M. Egerstedt, Graph Theoretic Methods in Multiagent Networks, Princeton University Press (2010)

[31] M. J. Osborne and A. Rubinstein, A Course in Game Theory, The MIT Press (1994)

[32] M. J. Osborne and A. Rubinstein, Bargaining and Markets, Academic Press, New York (1990)

[33] J. M. Ostroy, The no-surplus condition as a characterization of perfectly competitive equilibrium, J. Economic Theory 22, 183-207 (1980) 
[34] J. M. Ostroy, A reformulation of the marginal productivity theory of distribution, Econometrica 52, 3, 599-530 (1984)

[35] R. T. Rockafellar, Convex Analysis, Princeton Univ. Press (1970)

[36] R. T. Rockafellar and J-B. Wets, Variational Analysis, Springer Verlag, Berlin (1998)

[37] A. E. Roth and M. O. Sotomayor, Two-sided matching: A study in game-theoretic modeling and analysis, Cambridge Univ. Press, New York (1990)

[38] A. Rubinstein and A. Wolinsky, Decentralized trading, strategic behavior and the Walrasian outcome, The Review of Economic Studies 57, 63-78 (1990)

[39] J. Rust and G. Hall, Middlemen versus market makers: a theory of competitive exchange, J. Political Economy 111, 2, 353-403 (2003)

[40] D. G. Saari, Iterative price mechanisms, Econometrica 53, 1117-1131 (1985)

[41] L. Shapley and M. Shubik, Trade using one commodity as a means of payment, Journal of Political Economy 85, 937-968 (1977)

[42] R. M. Starr, The structure of exchange in barter and monetary economies, The Quarterly Journal of Economics 86, 2, 290-302 (1972)

[43] V. L. Smith, Microeconomic systems as an experimental science, American Economic Review 72, 5, 923-55 (1982)

[44] L. Tesfatsion and K. L. Judd, Handbook of Computational Economics II: Agent-Based Computational Economics, North-Holland (2006)

[45] A. Wilhite, Bilateral trade and "small-world" networks, Computational Economics 18, 49-64 (2001)

[46] M. H. Wooders, Equivalence of games and markets, Econometrica 62, 5,1141-1160 (1994) 